\title{
Association between Physical Fitness and Cardiometabolic Risk of Children and Adolescents in Korea
}

\author{
Hyun-Suk Lee', Won-Wook Jeong', Yu-Jeong Choi', Young-Gyun Seo' , Hye-Mi Noh', Hong-Ji Song', Yu-Jin Paek', \\ Yoon-Myung Kim², Hyun-Jung Lim ${ }^{3}$, Hye-Ja Lee ${ }^{4}$, Han-Byul Jang ${ }^{4}$, Sang-lck Park ${ }^{4}$, Kyung Hee Park ${ }^{1, *}$ \\ 'Department of Family Medicine, Hallym University Sacred Heart Hospital, Anyang, Korea \\ ${ }^{2}$ University College, Yonsei University International Campus, Incheon, Korea \\ ${ }^{3}$ Department of Medical Nutrition, Kyung Hee University, Yongin, Korea \\ ${ }^{4}$ Center for Biomedical Sciences, Korea National Institute of Health, Cheongju, Korea
}

Background: This study aimed to investigate the association between physical fitness and cardiometabolic health of Korean children and adolescents.

Methods: In total, 168 participants (89 boys and 79 girls) aged 10-16 years were recruited for the Intervention for Childhood and Adolescent Obesity via Activity and Nutrition Study in 2016. The subjects were categorized into two groups using the definition of metabolic syndrome by the International Diabetes Federation: metabolically unhealthy (with at least two of the five criteria) and healthy groups (with less than one criterion). Correlation analysis of the participants' general characteristics was performed. Odds ratios (ORs) of physical fitness for cardiometabolic risk were evaluated via logistic regression.

Results: Metabolically unhealthy children showed greater weight, height, and body mass index, higher Children's Depression Inventory score, and longer screen time than did the metabolically healthy children. Metabolically healthy children showed greater upper and lower extremity muscular strength than did the metabolically unhealthy children $(\mathrm{P}=0.04$ and $\mathrm{P}<0.001$, respectively). In the multiple logistic regression analysis, lower extremity muscle strength was inversely related to the clustered cardiometabolic risk of the children and adolescents with or without adjustment for confounders (OR, 4.32; 95\% confidence interval [CI], 1.87-9.97; OR, 7.64; 95\% CI, 1.5537.74 , respectively).

Conclusion: Physical fitness, especially lower extremity muscle strength, is significantly inversely associated with individual and clustered cardiometabolic risks in Korean children and adolescents.

Keywords: Cardiometabolic Risk; Physical Fitness; Cardiorespiratory Fitness; Child; Adolescent

Received: July 5, 2017, Revised: August 12, 2017, Accepted: August 29, 2017

*Corresponding Author: Kyung Hee Park https://orcid.org/0000-0001-9806-0076

Tel: +82-31-380-3805, Fax: +82-31-380-1782, E-mail: beloved920@gmail.com 


\section{INTRODUCTION}

Worldwide, cardiovascular diseases (CVDs) are the leading cause of both global mortality and disability. ${ }^{1)}$ Because the onset of atherosclerosis seems to occur in early childhood, it is currently recognized that CVDs are partly a pediatric problem. ${ }^{2)}$

Several risk factors for CVDs have been identified in children and adolescents, such as abdominal obesity, elevated blood pressure, insulin resistance, elevated triglyceride (TG) levels, and reduced highdensity lipoprotein (HDL) cholesterol levels. ${ }^{3)}$ These cardiometabolic risk factors during childhood tend to track into adulthood. ${ }^{4)}$ Accordingly, a global strategy based on knowledge of the importance of risk factors for CVDs for effective prevention is needed. ${ }^{5)}$

A higher level of cardiorespiratory fitness and muscle strength independently reduce the risk of cardiometabolic risk in adults. ${ }^{6)}$ Further, several studies on physical fitness and health in childhood and adolescence have been conducted in the last years. ${ }^{7)}$

Earlier studies suggest that a high level of cardiorespiratory fitness is associated with a healthier cardiovascular profile during childhood, ${ }^{8)}$ and muscle fitness has been recognized in the prevention of chronic diseases. ${ }^{9)}$ However, most of these preceding studies had been conducted predominantly in Western countries, and to our knowledge, there are a limited number of studies conducted on Asian children.

The primary aim of this study was to investigate the association between physical fitness and clustered cardiometabolic risk factors in Korean children and adolescents.

\section{METHODS}

\section{Subjects}

We conducted a cross-sectional analysis of the cardiometabolic health status of children and adolescents using data from the Intervention for Childhood and Adolescent Obesity via Activity and Nutrition (ICAAN) Study. The ICAAN Study is an intensive multidisciplinary intervention program including nutritional education, exercise education, and behavioral modification for children and adolescents with predominantly severe obesity in Korea. In this study, we used baseline data from 289 subjects aged 6-16 years who enrolled in the 16-week pilot study to test the feasibility of the ICAAN program. Children and adolescents without obesity were also included as active controls. Finally, a total of 168 participants (89 boys and 79 girls) were included in this study after excluding 121 participants who were younger than 10 years and whose data, such as laboratory test findings, survey data, and physical fitness test findings, were missing.

All subjects and their parents provided informed consents before participation in this study. All study protocols were approved by institutional review board and ethics committee in Hallym University Sacred Heart Hospital (IRB approval no., 2015-I134).

\section{Definition of Cardiometabolic Health Status}

To investigate the clustered cardiometabolic risk factors, we used the definition of metabolic syndrome by the International Diabetes Federation (IDF) in 2007. ${ }^{10,11)}$ We defined the metabolically unhealthy group (MUG) as children and adolescents having at least two of the following five criteria: (1) waist circumference (WC) of $\geq 90$ th percentile, (2) TG level of $\geq 150 \mathrm{mg} / \mathrm{dL}$, (3) HDL cholesterol level of $\leq 40 \mathrm{mg} / \mathrm{dL}$, (4) systolic or diastolic blood pressure (SBP or DBP, respectively) of $\geq 90$ th percentile, and (5) fasting plasma glucose level of $\geq 100 \mathrm{mg} / \mathrm{dL}$. The cutoff values of the WC and blood pressure were based on the 2007 Korean growth charts for children and adolescents. ${ }^{12)}$ The rest of the participants who did not meet the criteria described above were classified into the metabolically healthy group (MHG).

\section{Clinical Variables}

The weight and height were measured using the body composite analyzer, InBody 720 (Biospace Co., Seoul, Korea). The WC was measured to the nearest $0.1 \mathrm{~cm}$ during exhalation at the midpoint between the lower border of the ribcage and the iliac crest using a measuring tape. The body mass index (BMI) was calculated as weight $(\mathrm{kg})$ divided by the square of height $\left(\mathrm{m}^{2}\right)$. Blood pressure was measured twice using the DINAMAP DPC100X-EN automated blood pressure monitor (GE Medical System Information Technologies, Milwaukee, WI, USA) while the subjects were resting in a seated position; the two measures for each participant were averaged for the analysis.

Laboratory tests were performed only for the subjects whose parents had agreed to the performance of blood tests and provided written informed consent in advance. After at least 10 hours of fasting, blood samples were collected from the antecubital vein. The HDL cholesterol, TG, fasting serum glucose, and serum insulin levels were analyzed.

The homeostatic model assessment of insulin resistance (HOMA-IR) score was used as a measure of insulin resistance. ${ }^{13)}$ It was calculated by multiplying the fasting insulin level $(\mu \mathrm{IU} / \mathrm{mL})$ by the fasting glucose level (mg/dL) divided by $405 .^{14)}$

We performed three tests to evaluate the level of physical fitness. The arm curl test was used to measure the upper extremity muscle strength. The participants sat on a chair, held a dumbbell ( $4 \mathrm{~kg}$ for boys; $2 \mathrm{~kg}$ for girls) in their dominant hand and curled their hands up and lowered them down through the full range of motion. We counted the number of repetitions that they could perform in 30 seconds. The wall squat test was used to measure the lower extremity muscle strength. The participants stood comfortably with their feet approximately shoulder width apart and their back against the wall. They were asked to maintain a $90^{\circ}$ angle between their hips and knees. The total time in seconds was recorded. The 3-minute step test was used to measure cardiorespiratory fitness. The participants alternated their steps to a 30-cm height step platform within 3 minutes. One step cycle comprised four stages in the metronome cadence set at the rate of 96 beats per minute. They stepped up to the platform with one foot (first stage), stepped up with the second foot (second stage), stepped down with one foot (third stage), and stepped down with the other foot (fourth stage). The total 3-minute post-exercise heart rate of the partic- 
ipants was recorded.

\section{Questionnaire-Based Survey}

Some information on the factors that may influence metabolic syndrome in children and adolescents was obtained from the structured questionnaire completed by the participants and their parents. These factors included screen time, parents' educational level, monthly household income, and Children's Depression Inventory (CDI) score. $^{15)}$

\section{Statistical Analysis}

All data in this study were analyzed using STATA ver. 14.0 (Stata Corp., College Station, TX, USA). Using the Shapiro-Wilk test, categorical and continuous variables were checked for normality. The $\chi^{2}$ test for parametric analysis and Fisher's exact test for nonparametric analysis were used for categorical variables. Continuous variables were analyzed using the t-test and Wilcoxon rank-sum test. Some variables, such as age, weight, CDI score, BMI, WC, DBP, HDL cholesterol level, TG level, fasting plasma glucose level, screen time, HOMA-IR score, and wall squat test findings, were analyzed using the nonparametric method because their distribution were skewed. Continuous data were expressed as means and standard deviations and categorical data as frequencies and percentages. Data in the nonparametric analysis were expressed as medians and interquartile ranges. Correlation analysis was used to examine the statistical relationship involving dependence between individual physical fitness components and cardiometabolic risks.

Logistic regression analysis was conducted to investigate the relationship between the independent components of physical fitness and clustered cardiometabolic risk factors in children and adolescents. All tests were two-sided, and the level of significance was set at $\mathrm{P}<0.05$.

\section{RESULTS}

The general characteristics of the study subjects are shown in Table 1. We divided all study subjects into two groups: MHG and MUG ( $\mathrm{n}=109$ and $n=59$, respectively) according to the modified IDF criteria for defining metabolic syndrome in children and adolescents. The MUG showed greater weight, height, and BMI, higher CDI score, and longer screen time than did the MHG. However, there were no significant differences in the parents' educational level $(\mathrm{P}=0.280)$ and monthly household income $(\mathrm{P}=0.601)$ between the two groups. The MHG showed greater upper and lower extremity muscle strength than did the MUG ( $\mathrm{P}=0.04$ and $\mathrm{P}<0.001$, respectively). However, there was no significant difference in the cardiorespiratory function.

Table 2 shows the correlation of the individual components of physical fitness with the single or clustered cardiometabolic risk factors. A

Table 1. General characteristics of the study subjects

\begin{tabular}{|c|c|c|c|}
\hline Characteristic & Metabolically healthy group $(n=109)$ & Metabolically unhealthy group $(n=59)$ & P-value* \\
\hline Age $(y)^{\dagger}$ & $11.07(10.70-11.89)$ & $12.49(10.97-14.35)$ & $<0.001$ \\
\hline Sex & & & 0.001 \\
\hline Boys & $47(43.1)$ & $42(71.2)$ & \\
\hline Girls & $62(56.9)$ & $17(28.8)$ & \\
\hline Weight $(\mathrm{kg})^{\dagger}$ & $44.4(37.0-62.0)$ & $79.2(63.2-86.9)$ & $<0.001$ \\
\hline Height (cm) & $148.54 \pm 8.25$ & $159.11 \pm 9.08$ & $<0.001$ \\
\hline Body mass index $\left(\mathrm{kg} / \mathrm{m}^{2}\right)^{\dagger}$ & $20.78(17.97-26.21)$ & $29.65(27.28-34.19)$ & $<0.001$ \\
\hline Children's Depression Inventory score ${ }^{\dagger}$ & $6.0(4.0-12.0)$ & $11.0(5.0-16.0)$ & 0.004 \\
\hline Screen time $(h)^{\dagger}$ & $3.0(1.0-5.0)$ & $5.0(3.0-6.3)$ & $<0.001$ \\
\hline Parents' educational level & & & 0.280 \\
\hline Low & $37(33.9)$ & $25(42.4)$ & \\
\hline High & $72(66.1)$ & $34(57.6)$ & \\
\hline Monthly household income ( $10^{4}$ Korean won/mo) & & & 0.601 \\
\hline $\operatorname{Low}(<300)$ & $19(17.4)$ & $14(23.7)$ & \\
\hline Middle (300-500) & $44(40.4)$ & $23(39.0)$ & \\
\hline High $(\geq 500)$ & $46(42.2)$ & $22(37.3)$ & \\
\hline Arm curl test (attempts) & $19.52 \pm 5.45$ & $17.59 \pm 6.32$ & 0.040 \\
\hline Wall squat test $(\mathrm{s})^{\dagger}$ & $65.0(39.0-90.0)$ & $35.0(20.0-60.0)$ & $<0.001$ \\
\hline 3-Minute step test (beats/min) & $134.40 \pm 20.03$ & $128.44 \pm 20.59$ & 0.070 \\
\hline Waist circumference $(\mathrm{cm})^{\dagger}$ & $71.9(63.0-85.4)$ & $95.0(90.5-102.2)$ & $<0.001$ \\
\hline Systolic blood pressure (mm Hg) & $110.06 \pm 12.23$ & $128.81 \pm 19.14$ & $<0.001$ \\
\hline Diastolic blood pressure $(\mathrm{mm} \mathrm{Hg})^{\dagger}$ & $63.8(58.5-70.0)$ & $69.5(64.5-75.0)$ & 0.001 \\
\hline Triglyceride level (mg/dL) ${ }^{\dagger}$ & $77.0(59.0-93.0)$ & $142.0(92.0-183.0)$ & $<0.001$ \\
\hline High-density lipoprotein cholesterol level $(\mathrm{mg} / \mathrm{dL})^{\dagger}$ & $57.0(49.0-66.0)$ & $41.0(37.0-52.0)$ & $<0.001$ \\
\hline Glucose level $(\mathrm{mg} / \mathrm{dL})^{\dagger}$ & $91.0(87.0-95.0)$ & $89.0(85.0-95.0)$ & 0.171 \\
\hline Homeostatic model assessment of insulin resistance score ${ }^{\dagger}$ & $2.57(1.43-3.89)$ & $4.84(3.82-8.19)$ & $<0.001$ \\
\hline
\end{tabular}

Values are presented as mean (interquartile range) for nonparametric analysis data, number (\%) for categorical variables, or mean \pm standard deviation for continuous variables. ${ }^{*}$ Analyzed using the t-test, chi-square test, or Wilcoxon rank-sum test. ${ }^{\dagger}$ Analyzed using the Wilcoxon rank-sum test and expressed as mean (interquartile range). 
Table 2. Correlation analysis between each type of physical fitness and single components of cardiometabolic risks

\begin{tabular}{|c|c|c|c|c|c|c|}
\hline \multirow{2}{*}{ Variable } & \multicolumn{2}{|c|}{ Arm curl test } & \multicolumn{2}{|c|}{ Wall squat test } & \multicolumn{2}{|c|}{ 3-Minute step test } \\
\hline & $r^{*}$ & P-value ${ }^{\dagger}$ & r & P-value ${ }^{\dagger}$ & r & P-value ${ }^{\dagger}$ \\
\hline Body mass index & -0.195 & 0.011 & -0.478 & $<0.001$ & -0.091 & 0.239 \\
\hline Waist circumference & -0.248 & 0.001 & -0.522 & $<0.001$ & -0.099 & 0.201 \\
\hline Systolic blood pressure & -0.218 & 0.005 & -0.338 & $<0.001$ & -0.031 & 0.693 \\
\hline High-density lipoprotein cholesterol level & 0.200 & 0.009 & 0.334 & $<0.001$ & 0.128 & 0.097 \\
\hline Triglyceride G level & -0.160 & 0.038 & -0.321 & $<0.001$ & 0.004 & 0.962 \\
\hline Fasting blood glucose level & 0.075 & 0.333 & 0.046 & 0.550 & 0.153 & 0.048 \\
\hline Homeostatic model assessment of insulin resistance score & -0.074 & 0.341 & -0.260 & $<0.001$ & 0.054 & 0.486 \\
\hline Age & -0.020 & 0.796 & -0.224 & 0.004 & -0.114 & 0.140 \\
\hline
\end{tabular}

*Pearson's correlation coefficient. ${ }^{\dagger}$ Analyzed using Pearson's correlation.

Table 3. Odds ratios of physical fitness for cardiometabolic risks

\begin{tabular}{|c|c|c|c|c|c|}
\hline Variable & Category & Unadjusted & Model 1 & Model 2 & Model 3 \\
\hline \multirow[t]{3}{*}{ Muscle fitness (upper extremity) } & Upper one-third & 1.00 (reference) & 1.00 (reference) & 1.00 (reference) & 1.00 (reference) \\
\hline & Lower two-third & $1.16(0.57-2.39)$ & $0.99(0.43-2.28)$ & $0.80(0.30-2.11)$ & $0.60(0.0 .17-2.06)$ \\
\hline & $P$-value & 0.676 & 0.978 & 0.655 & 0.417 \\
\hline \multirow[t]{3}{*}{ Muscle fitness (lower extremity) } & Upper one-third & 1.00 (reference) & 1.00 (reference) & 1.00 (reference) & 1.00 (reference) \\
\hline & Lower two-third & $4.32(1.87-9.97)$ & $6.81(2.47-18.72)$ & $3.85(1.25-11.80)$ & $7.64(1.55-37.74)$ \\
\hline & P-value & 0.001 & $<0.001$ & 0.019 & 0.013 \\
\hline \multirow[t]{3}{*}{ Cardiorespiratory fitness } & Upper one-third & 1.00 (reference) & 1.00 (reference) & 1.00 (reference) & 1.00 (reference) \\
\hline & Lower two-third & $1.11(0.58-2.10)$ & $1.03(0.49-2.19)$ & $0.79(0.34-1.86)$ & $0.58(0.19-1.77)$ \\
\hline & P-value & 0.760 & 0.930 & 0.591 & 0.338 \\
\hline
\end{tabular}

Values are presented as odds ratio (95\% confidence interval). Model 1: adjusted for age and sex; model 2: adjusted for age, sex, and BMl; model 3: adjusted for age, sex, BMI, waist circumference, systolic blood pressure, high-density lipoprotein cholesterol level, homeostatic model assessment of insulin resistance score, screen time, monthly household income, and parents' educational level.

BMl, body mass index.

negative correlation was observed for the BMI, WC, SBP, and TG level; the only positive correlation was observed between the HDL cholesterol level and upper extremity muscle strength $(\mathrm{P}<0.05)$. However, there was no significant correlation with the fasting plasma glucose level and HOMA-IR score. A negative correlation was observed between lower extremity muscle strength and most of the cardiometabolic risk factors, including age $(\mathrm{P}=0.004)$. Conversely, a positive correlation with the HDL cholesterol level was observed $(\mathrm{P}<0.001)$; however, there was no significant correlation with the fasting blood glucose level. A negative correlation was observed only between the fasting blood glucose level and cardiorespiratory function $(\mathrm{P}=0.048)$, and there was no significant association between the rest of the cardiometabolic risk factors and cardiorespiratory function.

Table 3 shows the odds ratios (ORs) of physical fitness for cardiometabolic risk after sequentially adjusting for age, sex, BMI, WC, SBP, HDL cholesterol level, HOMA-IR score, screen time, monthly household income, and parents' educational level. Considering that physical fitness is particularly relevant to age and sex, we divided all the subjects by age (10-12 years or above) and by sex (boys or girls). Each type of physical fitness was divided into upper one-third and lower twothird using tertiles by age-sex group; we then merged them into each level of fitness (upper one-third and lower two-third). Upper extremity muscle strength and cardiorespiratory fitness were not associated with the clustered cardiometabolic risks regardless of any adjustment.
However, even when no adjustments were made, a significant association was observed between lower extremity muscle fitness and cardiometabolic risk (OR, 4.37; 95\% confidence interval [CI], 1.87-9.97). After adjusting for age, sex, WC, SBP, HDL cholesterol level, HOMA-IR score, screen time, monthly household income, and parents' educational level, low level of lower extremity muscle fitness showed a stronger association with the cardiometabolic risks (OR, 7.64; 95\% CI, 1.55-37.74).

\section{DISCUSSION}

This study was designed to investigate the association between the types of physical fitness and cardiometabolic risks in Korean children and adolescents. We found that the lower extremity muscle strength is inversely related to the clustered cardiometabolic risk of children and adolescents in Korea. In addition, there was a stronger association between lower extremity muscle strength and individual cardiometabolic risk factors than between the upper extremity muscle strength and cardiorespiratory fitness. In adults, it is widely known that a high level of muscle fitness reduces the risks for CVDs by decreasing the blood pressure, TG level, ${ }^{16)}$ and incidence of abdominal obesity ${ }^{17)}$ or increasing the HDL cholesterol level ${ }^{18)}$ and insulin sensitivity. ${ }^{19)}$ However, differing results on the association between physical fitness and cardiometabolic risks in children and adolescents have been reported. Although our results are not consistent with the following findings, some 
authors found the association of cardiorespiratory fitness with clustered metabolic risks to be slightly stronger than that with muscle strength. ${ }^{20)}$ Other researchers suggested that muscle strength, especially that of the lower extremity, shows a stronger association with cardiometabolic risks, ${ }^{21)}$ and our finding was consistent with this conclusion. One possible explanation for this is the increase in fat-free mass. In obesity, a greater amount of adipose tissue is gained. Fat-free mass also increases as the amount of adiposity increases, and this supports the extra load. ${ }^{22)}$ For this reason, children with obesity tend to perform upper extremity muscle exercises more easily and their lower extremity muscle strength tends to be lower. ${ }^{23)}$

In the present study, we also observed that insulin resistance measured using the HOMA-IR score was negatively related to the lower extremity muscle strength. Insulin resistance is characterized by a decrease in the ability of insulin to stimulate the use of glucose by the muscles and adipose tissue and to inhibit glucose production and output. ${ }^{24,25)}$ It can be influenced by both genetic and environmental factors, including obesity and sedentary lifestyle. ${ }^{26)}$ Obesity-mediated insulin resistance can result in some complications, such as dyslipidemia, impaired glucose tolerance or type 2 diabetes, hepatic steatosis, early atherosclerosis, and polycystic ovary syndrome. ${ }^{27)}$ Eventually, insulin resistance in childhood can track into adulthood. ${ }^{28)}$ Since it is not clear whether these effects are due to changes in muscle metabolism, insulin regulation, counterregulatory hormones, or adipose tissue induced by exercise, further study on this area is needed.

We also found no significant association with a low socioeconomic status (SES), including parents' educational level and monthly household income. Some previous studies have shown that the SES in childhood is inversely related to cardiometabolic risk factors and vascular structure and function. ${ }^{29)}$ The discrepancy in these results may originate from cultural differences. The proportion of the population with tertiary education among South Koreans aged 25 to 64 is the highest among the Organization for Economic Cooperation and Development countries. ${ }^{30)}$ The rate of tertiary educational attainment in the parents or sponsors of the subjects in this study was $63.1 \%$ (106 out of 168), which may act as one of the confounders.

Our study has three limitations. First, the study sample cannot represent all children and adolescents of our country because of the small sample size and the limited available region. Second, owing to the cross-sectional nature of the study, we could not determine the causality between increase in physical fitness and change in clustered or individual cardiometabolic risk factors. Third, sex and age could act as confounders; however, no definite standard reference values of each physical fitness are available. Therefore, we attempted to calibrate these variables using statistical analyses stratified by sex and age and adjusting for multiple confounding factors, including sex and age.

Despite these limitations, this is the first study that investigated the relationship between cardiometabolic risks and physical fitness in Korean children and adolescents to the best of our knowledge. In conclusion, physical fitness, especially lower extremity muscle strength, is significantly inversely associated with individual and clustered cardio- metabolic risks in Korean children and adolescents. Therefore, lower extremity muscle fitness can be an important predictor of cardiometabolic risks in children and adolescents. Further studies are needed to investigate the cardiometabolic change in relation to increase in physical fitness.

\section{CONFLICT OF INTEREST}

No potential conflict of interest relevant to this article was reported.

\section{ACKNOWLEDGMENTS}

This research was supported by a fund (2015-ER6401-00) from the Research of Korea Centers for Disease Control and Prevention.

\section{ORCID}

Hyun-Suk Lee: https://orcid.org/0000-0003-0762-8995

Won-Wook Jeong: https://orcid.org/0000-0002-1582-4526

Yu-Jeong Choi: https://orcid.org/0000-0001-5463-4509

Young-Gyun Seo: https://orcid.org/0000-0001-8294-1741

Hye-Mi Noh: https://orcid.org/0000-0003-2112-1613

Hong-Ji Song: https://orcid.org/0000-0002-3563-9504

Yu-Jin Paek: https://orcid.org/0000-0001-9573-8849

Yoon-Myung Kim: https://orcid.org/0000-0002-6582-1446

Hyun-Jung Lim: https://orcid.org/0000-0001-7632-7315

Hye-Ja Lee: https://orcid.org/0000-0002-7120-1809

Han-Byul Jang: https://orcid.org/0000-0002-5826-1698

Sang-Ick Park: https://orcid.org/0000-0003-4115-9117

Kyung Hee Park: https://orcid.org/0000-0001-9806-0076

\section{REFERENCES}

1. Murray CJ, Lopez AD. Evidence-based health policy: lessons from the Global Burden of Disease Study. Science 1996;274:740-3.

2. Kavey RE, Daniels SR, Lauer RM, Atkins DL, Hayman LL, Taubert K, et al. American Heart Association guidelines for primary prevention of atherosclerotic cardiovascular disease beginning in childhood. Circulation 2003;107:1562-6.

3. Saland JM. Update on the metabolic syndrome in children. Curr Opin Pediatr 2007;19:183-91.

4. Raitakari OT, Juonala M, Kahonen M, Taittonen L, Laitinen T, MakiTorkko N, et al. Cardiovascular risk factors in childhood and carotid artery intima-media thickness in adulthood: the Cardiovascular Risk in Young Finns Study. JAMA 2003;290:2277-83.

5. Yusuf S, Hawken S, Ounpuu S, Dans T, Avezum A, Lanas F, et al. Effect of potentially modifiable risk factors associated with myocardial infarction in 52 countries (the INTERHEART study): case-control study. Lancet 2004;364:937-52.

6. LaMonte MJ, Barlow CE, Jurca R, Kampert JB, Church TS, Blair SN. Cardiorespiratory fitness is inversely associated with the incidence of metabolic syndrome: a prospective study of men and women. Circulation 2005;112:505-12. 
7. Artero EG, Ruiz JR, Ortega FB, Espana-Romero V, Vicente-Rodriguez G, Molnar D, et al. Muscular and cardiorespiratory fitness are independently associated with metabolic risk in adolescents: the HELENA study. Pediatr Diabetes 2011;12:704-12.

8. Brage S, Wedderkopp N, Ekelund U, Franks PW, Wareham NJ, Andersen LB, et al. Features of the metabolic syndrome are associated with objectively measured physical activity and fitness in Danish children: the European Youth Heart Study (EYHS). Diabetes Care 2004;27:21418.

9. Stump CS, Henriksen EJ, Wei Y, Sowers JR. The metabolic syndrome: role of skeletal muscle metabolism. Ann Med 2006;38:389-402.

10. Kelishadi R, Razaghi EM, Gouya MM, Ardalan G, Gheiratmand R, Delavari A, et al. Association of physical activity and the metabolic syndrome in children and adolescents: CASPIAN Study. Horm Res 2007;67:46-52.

11. Zimmet P, Alberti KG, Kaufman F, Tajima N, Silink M, Arslanian S, et al. The metabolic syndrome in children and adolescents: an IDF consensus report. Pediatr Diabetes 2007;8:299-306.

12. Moon JS, Lee SY, Nam CM, Choi JM, Choe BK, Seo JW, et al. 2007 Korean national growth charts: review of developmental process and an outlook. Korean J Pediatr 2008;51:1-25.

13. Matthews DR, Hosker JP, Rudenski AS, Naylor BA, Treacher DF, Turner RC. Homeostasis model assessment: insulin resistance and beta-cell function from fasting plasma glucose and insulin concentrations in man. Diabetologia 1985;28:412-9.

14. Peplies J, Jimenez-Pavon D, Savva SC, Buck C, Gunther K, Fraterman A, et al. Percentiles of fasting serum insulin, glucose, HbAlc and HOMA-IR in pre-pubertal normal weight European children from the IDEFICS cohort. Int J Obes (Lond) 2014;38 Suppl 2:S39-47.

15. Helsel WJ, Matson JL. The assessment of depression in children: the internal structure of the Child Depression Inventory (CDI). Behav Res Ther 1984;22:289-98.

16. Kohl HW 3rd, Gordon NF, Scott CB, Vaandrager H, Blair SN. Musculoskeletal strength and serum lipid levels in men and women. Med Sci Sports Exerc 1992;24:1080-7.

17. Treuth MS, Ryan AS, Pratley RE, Rubin MA, Miller JP, Nicklas BJ, et al. Effects of strength training on total and regional body composition in older men. J Appl Physiol (1985) 1994;77:614-20.

18. Hurley BF, Hagberg JM, Goldberg AP, Seals DR, Ehsani AA, Brennan $\mathrm{RE}$, et al. Resistive training can reduce coronary risk factors without altering VO2max or percent body fat. Med Sci Sports Exerc 1988;20:1504.

19. Ishii T, Yamakita T, Sato T, Tanaka S, Fujii S. Resistance training improves insulin sensitivity in NIDDM subjects without altering maximal oxygen uptake. Diabetes Care 1998;21:1353-5.

20. Zaqout M, Michels N, Bammann K, Ahrens W, Sprengeler O, Molnar $\mathrm{D}$, et al. Influence of physical fitness on cardio-metabolic risk factors in European children. The IDEFICS study. Int J Obes (Lond) 2016;40: 1119-25.

21. Benson AC, Torode ME, Singh MA. Muscular strength and cardiorespiratory fitness is associated with higher insulin sensitivity in children and adolescents. Int J Pediatr Obes 2006;1:222-31.

22. Forbes GB. Lean body mass and fat in obese children. Pediatrics 1964;34:308-14.

23. Bar-Or O. Physical activity and physical training in childhood obesity. J Sports Med Phys Fitness 1993;33:323-9.

24. Matthaei S, Stumvoll M, Kellerer M, Haring HU. Pathophysiology and pharmacological treatment of insulin resistance. Endocr Rev 2000;21:585-618.

25. Chiarelli F, Marcovecchio ML. Insulin resistance and obesity in childhood. Eur J Endocrinol 2008;159 Suppl 1:S67-74.

26. Liu L, Hironaka K, Pihoker C. Type 2 diabetes in youth. Curr Probl Pediatr Adolesc Health Care 2004;34:254-72.

27. Eckel RH, Grundy SM, Zimmet PZ. The metabolic syndrome. Lancet 2005;365:1415-28.

28. Sinaiko AR, Steinberger J, Moran A, Hong CP, Prineas RJ, Jacobs DR Jr. Influence of insulin resistance and body mass index at age 13 on systolic blood pressure, triglycerides, and high-density lipoprotein cholesterol at age 19. Hypertension 2006;48:730-6.

29. Arslanian S, Suprasongsin C. Insulin sensitivity, lipids, and body composition in childhood: is "syndrome X" present? J Clin Endocrinol Metab 1996;81:1058-62.

30. Organization for Economic Cooperation and Development. Education at a glance 2014: OECD indicators. Paris: OECD Publishing; 2014. 